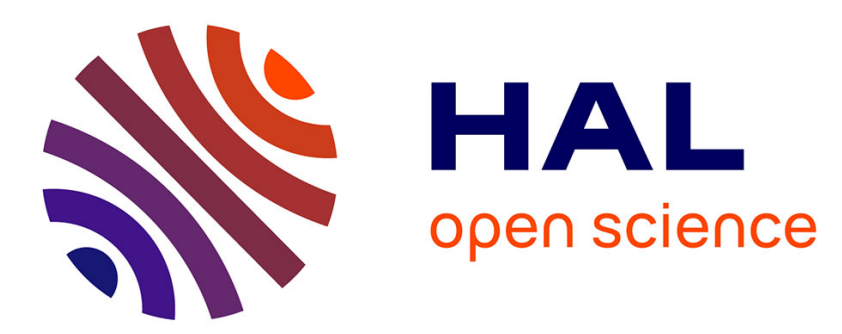

\title{
Evolution hydrogéologique de l'hydrosystème karstique Cesse-Pouzols (Minervois, France) au cours du quaternaire
}

Alexandre Nou, Séverin Pistre, Christelle Batiot-Guilhe, Valérie

Borrell-estupina

\section{To cite this version:}

Alexandre Nou, Séverin Pistre, Christelle Batiot-Guilhe, Valérie Borrell-estupina. Evolution hydrogéologique de l'hydrosystème karstique Cesse-Pouzols (Minervois, France) au cours du quaternaire. Quaternaire, 2013, vol. 24/1, pp.25-34. 10.4000/quaternaire.6461 . hal-02410278

\section{HAL Id: hal-02410278 \\ https://hal.science/hal-02410278}

Submitted on 3 Sep 2021

HAL is a multi-disciplinary open access archive for the deposit and dissemination of scientific research documents, whether they are published or not. The documents may come from teaching and research institutions in France or abroad, or from public or private research centers.
L'archive ouverte pluridisciplinaire HAL, est destinée au dépôt et à la diffusion de documents scientifiques de niveau recherche, publiés ou non, émanant des établissements d'enseignement et de recherche français ou étrangers, des laboratoires publics ou privés. 


\title{
Quaternaire
}

vol. 24/1 (2013)

Volume 24 Numéro 1

Alexandre Nou, Séverin Pistre, Christelle Batiot-Guilhe et Valérie BorrellEstupina

\section{Évolution hydrogéologique de l'hydrosystème karstique Cesse- Pouzols (Minervois, France) au cours du Quaternaire}

\author{
Avertissement \\ Le contenu de ce site relève de la législation française sur la propriété intellectuelle et est la propriété exclusive de \\ l'éditeur. \\ Les œuvres figurant sur ce site peuvent être consultées et reproduites sur un support papier ou numérique sous \\ réserve qu'elles soient strictement réservées à un usage soit personnel, soit scientifique ou pédagogique excluant \\ toute exploitation commerciale. La reproduction devra obligatoirement mentionner l'éditeur, le nom de la revue, \\ l'auteur et la référence du document. \\ Toute autre reproduction est interdite sauf accord préalable de l'éditeur, en dehors des cas prévus par la législation \\ en vigueur en France.
}

\section{revues.org}

Revues.org est un portail de revues en sciences humaines et sociales développé par le Cléo, Centre pour l'édition électronique ouverte (CNRS, EHESS, UP, UAPV).

\section{Référence électronique}

Alexandre Nou, Séverin Pistre, Christelle Batiot-Guilhe et Valérie Borrell-Estupina, «Évolution hydrogéologique de l'hydrosystème karstique Cesse-Pouzols (Minervois, France) au cours du Quaternaire », Quaternaire [En ligne], vol. 24/1 | 2013, mis en ligne le 01 mars 2016, consulté le 02 septembre 2016. URL : http:// quaternaire.revues.org/6461; DOI : 10.4000/quaternaire.6461

Éditeur : Association Française pour l'Étude du Quaternaire http://quaternaire.revues.org

http://www.revues.org

Document accessible en ligne sur : http://quaternaire.revues.org/6461

Ce document est le fac-similé de l'édition papier.

(c) Tous droits réservés 


\title{
ÉVOLUTION HYDROGÉOLOGIQUE DE L'HYDROSYSTÈME KARSTIQUE CESSE-POUZOLS (MINERVOIS, FRANCE) AU COURS DU QUATERNAIRE
}

\author{
Alexandre NOU ${ }^{1}$, Séverin PISTRE ${ }^{1}$, Christelle BATIOT-GUILHE ${ }^{1}$ \\ \& Valérie BORRELL-ESTUPINA ${ }^{1}$
}

\begin{abstract}
RÉSUMÉ
L'hydrosystème karstique Cesse-Pouzols, en cours d'exploitation, se situe dans le Languedoc-Roussillon, dans le Minervois. La géologie de la zone est complexe et a suscité un intérêt pluridisciplinaire (géologie, hydrogéologie et géomorphologie), sans pour autant apporter un schéma de fonctionnement hydrogéologique global. À partir de la réinterprétation des travaux antérieurs guidée par de nouvelles observations en particulier sur les directions de fracturation et de drains karstiques, son évolution géomorphologique et hydrogéologique au cours du Quaternaire a pu être établie. Sur la base de cette évolution complexe et polyphasée de l'hydrosystème Cesse-Pouzols, un schéma de fonctionnement hydrogéologique cohérent avec son comportement hydrodynamique actuel est proposé, afin d'améliorer les connaissances et la gestion de cet hydrosystème.
\end{abstract}

Mots-clés: Quaternaire, évolution hydrogéologique, karst, Minervois, Cesse

\section{ABSTRACT}

HYDROGEOLOGIC EVOLUTION OF THE CESSE-POUZOLS KARSTIC HYDROSYSTEM (MINERVOIS, FRANCE) DURING THE QUATERNARY

The Cesse-Pouzols karst hydrosystem, currently exploited, is located in the Languedoc-Roussillon in the Minervois area. The complex geology of this area arose a pluridisciplinary interest (geology, hydrogeology and geomorphology), without bringing a global hydrogeologic functioning. From the analysis between precedent works and new observations about the direction of fractures and karstic conduits, the geomorphology and hydrogeology evolution during Quaternary was performed. Based on this complex and polyphased evolution of the hydrosystem, a hydrogeologic functioning scheme is proposed, in order to improve the knowledge and the management of this hydrosystem.

Keywords: Quaternary, hydrogeological evolution, karst, Minervois, Cesse

\section{1 - INTRODUCTION}

Le bassin versant karstique méditerranéen du Minervois et plus particulièrement l'hydrosystème CessePouzols, se situe dans le Languedoc-Roussillon à cheval sur les départements de l'Aude et de l'Hérault. Cet hydrosystème, en cours d'exploitation pour sa ressource souterraine, se positionne à la jonction du versant sud de la Montagne Noire, du chaînon de Saint-Chinian et de la terminaison nord de la nappe des Corbières. La géologie complexe, la taille moyenne du bassin versant $\left(275 \mathrm{~km}^{2}\right)$ ainsi que les relations fortes entre eau de surface et eau souterraine ont suscité un intérêt scientifique i) hydrogéologique (Guerret, 1951; Orengo, 1969; Yvroux, 2001) ii) géologique (Gèze, 1951; Arthaud, 1964; Chaigne, 1964; Plaziat, 1972; Bertolini, 1980; Genna, 1989; Berger, 1990) et iii) géomorphologique (Le Coz, 1967; Ambert,
1976; Ambert et al., 1977; Cornet, 1977; Larue, 2001 ; Genna \& Capdeville, 2007; Larue, 2008). Ces différents travaux n'ont pas permis d'aboutir à un schéma de fonctionnement hydrogéologique global satisfaisant, pourtant nécessaire à l'amélioration de la compréhension et de l'exploitation de cet hydrosystème. Dans un premier temps, le contexte hydrogéologique régional sera présenté ainsi que les nouvelles observations sur la fracturation et l'orientation des drains karstiques. Dans un deuxième temps, à la lumière des nouvelles observations issues de l'analyse de l'inventaire spéléologique et de mesures tectoniques de terrain, les travaux antérieurs sont reconsidérés de manière conjointe et pluridisciplinaire. Dans le cadre de la reconstitution de l'histoire récente du bassin versant, le travail mené s'est attaché à mettre en cohérence l'ensemble des observations relatives à la structure du réservoir karstique. Finalement, un scénario d'évolu-

\footnotetext{
${ }^{1}$ HydroSciences Montpellier, UMR 5569, Université Montpellier II, 300 avenue Émile Jeanbrau, F-34090 MONTPELLIER.

Courriel:nou@msem.univ-montp2.fr, pistre@msem.univ-montp2.fr
} 
tion hydrogéologique au cours du Quaternaire, compatible avec le comportement hydrodynamique actuel de l'aquifère, sera construit à partir de cette synthèse. En conclusion, un schéma de fonctionnement hydrogéologique global sera proposé.

\section{2 - CONTEXTE HYDROGÉOLOGIQUE RÉGIONAL}

La lithologie du bassin versant de la Cesse débute par le socle primaire du versant sud de la Montagne Noire avec des terrains allant du Cambrien au Dévonien, principalement constitués de calcaires dolomitiques et de schistes (Bertolini, 1980; Berger, 1990). Puis, sur ces terrains de l'ère primaire, viennent mordre en discordance des terrains cénozoïques constitués des calcaires marins à Alvéolines (Ilerdien) sur lesquels se sont déposées les molasses de Carcassonne composées des calcaires de Ventenac (Cuisien inférieur), de la formation grésomarneuse d'Assignan (Cuisien supérieur), des calcaires d'Agel (Lutétien) et des grès d'Aigne (Bartonien) (Genna \& Capdeville, 2007). Ces terrains ont été affectés par la tectonique hercynienne pour les terrains primaires et par la tectonique pyrénéenne pour les terrains tertiaires qui se structurent en une série d'anticlinaux et de synclinaux d'axe nord-est/sud-ouest (fig. 1). Quelques failles majeures suivent cette direction. Héritées de l'épisode hercynien, elles ont contribué à la déformation compressive de l'avant pays pyrénéen et ont pu rejouer à l'OligoMiocène, au moment de l'ouverture du Golfe du Lion comme la faille de Sainte-Valière.

Le territoire karstique du Minervois se compose d'un aquifère karstique principal localisé dans des terrains éocènes (aquifère de Pouzols) et de la rivière temporaire de la Cesse. Cet ensemble forme le bassin versant karstique binaire Cesse-Pouzols. Dans son cours ouest-est, la Cesse s'assèche la majeure partie de l'année et présente de nombreuses pertes et/ou résurgences selon l'état de l'aquifère et le régime de la rivière. Cette relation pertes/ résurgences traduit la présence de fortes interactions entre eau de surface et eau souterraine. Ces interactions, peuvent être concentrées (secteur de Fauzan par exemple) ou diffuses le long du parcours de la rivière à travers les causses de Minerve (entre le secteur de Fauzan et le village de Minerve par exemple).

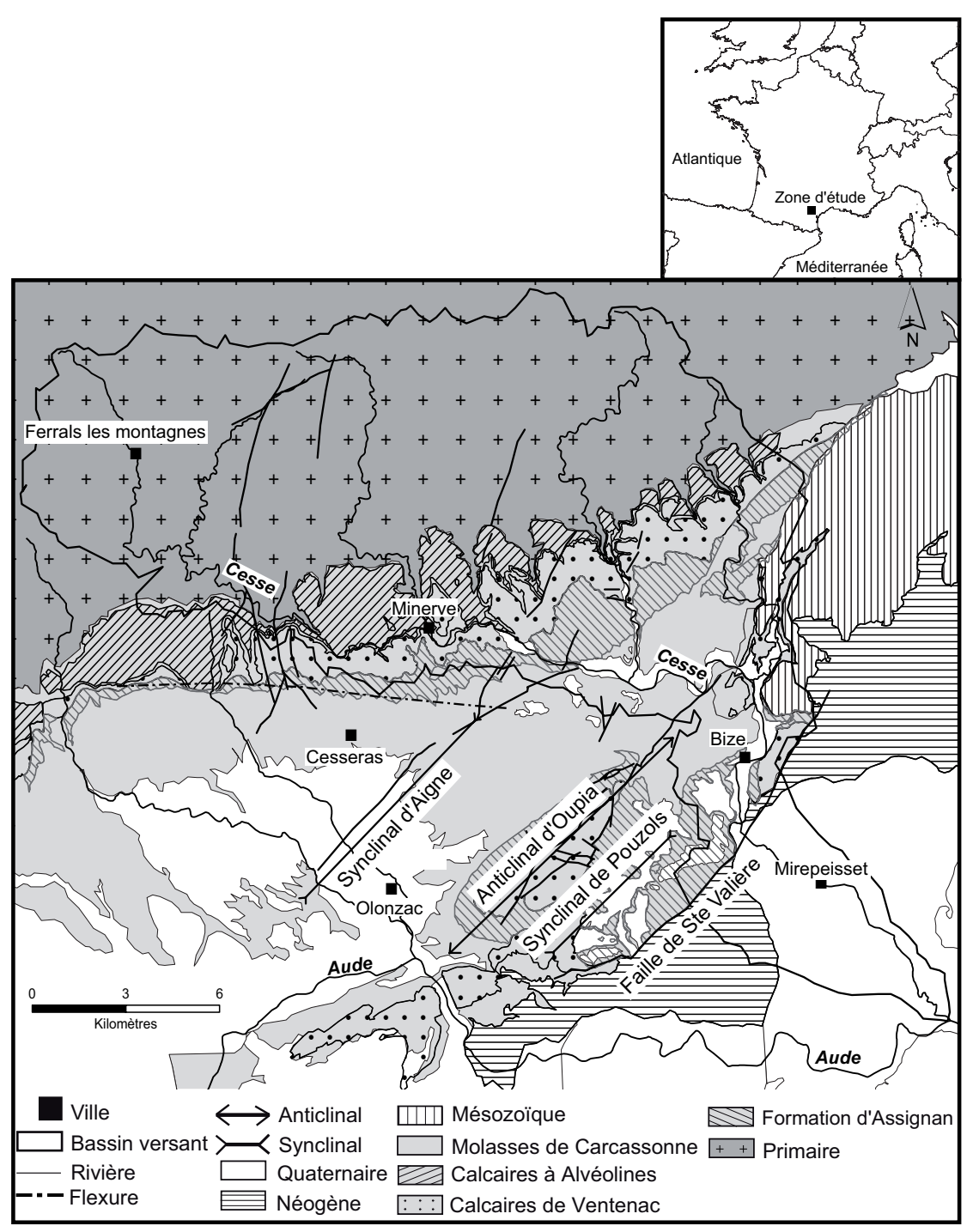

Fig. 1 : Localisation et schéma structural de l'hydrosystème Cesse-Pouzols.

Fig. 1: Location and structural plan of the Cesse-Pouzols hydrosystem. 
L'analyse de photos aériennes couplée à un relevé sur site de la fracturation ont permis d'établir la distribution de la fracturation à l'échelle de l'hydrosystème Cesse-Pouzols. Puis l'orientation de la fracturation a été confrontée à l'orientation des drains karstiques relevés à partir des plans de 46 grottes regroupées sur 8 secteurs du bassin versant d'après l'inventaire karstique réalisé récemment. Cet inventaire karstique concerne des grottes aujourd'hui considérées comme fossiles, mais aussi des réseaux actifs du secteur de Cesseras-Fauzan et de Minerve-Mayranne (fig. 2). Dans la partie la plus occidentale et méridionale du bassin (secteur de Cesseras-Fauzan et de RieussecBoisset), l'orientation des cavités explorées suit globalement les directions de fracturation N140-160 et N60-80 dont l'origine est hercynienne (Bertolini, 1980). Sur le secteur de Fauzan s'observe également un développement karstique suivant une direction N100-110, dans des grottes qui se sont formées au contact des terrains primaires et tertiaires (ex: grotte d'Aldène). Les directions N100-110 peuvent être attribuées à la fracturation pyrénéenne dont l'axe de compression est globalement perpendiculaire. Au centre du bassin, dans les secteurs de Minerve et de La Caunette, le développement karstique se fait selon les directions N20-40 et N140-160. Ces deux familles de drains suivent les directions de fracturations relevées dans ces secteurs qui sont associées aux tectoniques hercynienne et pyrénéenne. Toutefois, il apparaît une autre famille de drains orientée N90-110 qui ne se retrouve pas dans les relevés de fracturation. Des réseaux parallèles à la Cesse (en rive droite), orientés N90-110, de quelques mètres de long sont explorables au niveau de La Caunette (observations spéléologiques). Le développement karstique orienté N90-110 se ferait donc le long des joints de stratification. Plus à l'Est, dans les secteurs du Tréménal et de Cailhol, les galeries des grottes se déve- loppent principalement selon une direction N20-40. Une deuxième direction, N90-110, se distingue clairement dans le secteur du Tréménal. Ici aussi la direction des conduits suit l'orientation de la fracturation. Le secteur de Saint Jean du Minervois, est caractérisé par un développement karstique qui suit les directions N140-160 et N20-40, correspondant aussi à une fracturation héritée des tectoniques hercynienne et pyrénéenne. Le secteur de Bize se situe dans l'arc de St-Chinian, en marge du bassin de la Cesse. Les galeries des grottes se développent selon une direction principale N140. Les directions N40 et N100 sont également représentées dans ce secteur mais aucune mesure de la fracturation n'a pu être effectuée dans ce secteur.

À l'échelle de l'hydrosystème, deux épisodes tectoniques majeurs sont présents: la tectonique hercynienne et la tectonique pyrénéenne. Sur tous les secteurs présentés ici, les directions préférentielles des conduits karstiques suivent les directions de la fracturation sauf pour le secteur de Minerve, et dans une moindre mesure le secteur de La Caunette où des drains karstiques se développent aussi dans les joints de stratification.

Il est donc proposé ici d'analyser d'un point de vue pluridisciplinaire les précédents travaux afin de proposer une évolution hydrogéologique au cours du Quaternaire en vue i) de proposer une explication à la présence de drains karstiques développés le long des joints de stratification dans le secteur de Minerve et ii) d'établir un schéma de fonctionnement hydrogéologique global cohérent de l'hydrosystème Cesse-Pouzols afin d'en améliorer la compréhension et l'exploitation. Il s'agit notamment, à la lumière des observations sur la karstification essentiellement fossile ou fonctionnelle et de données hydrogéologiques actuelles, d'élaborer un schéma d'évolution de l'ensemble du bassin versant.

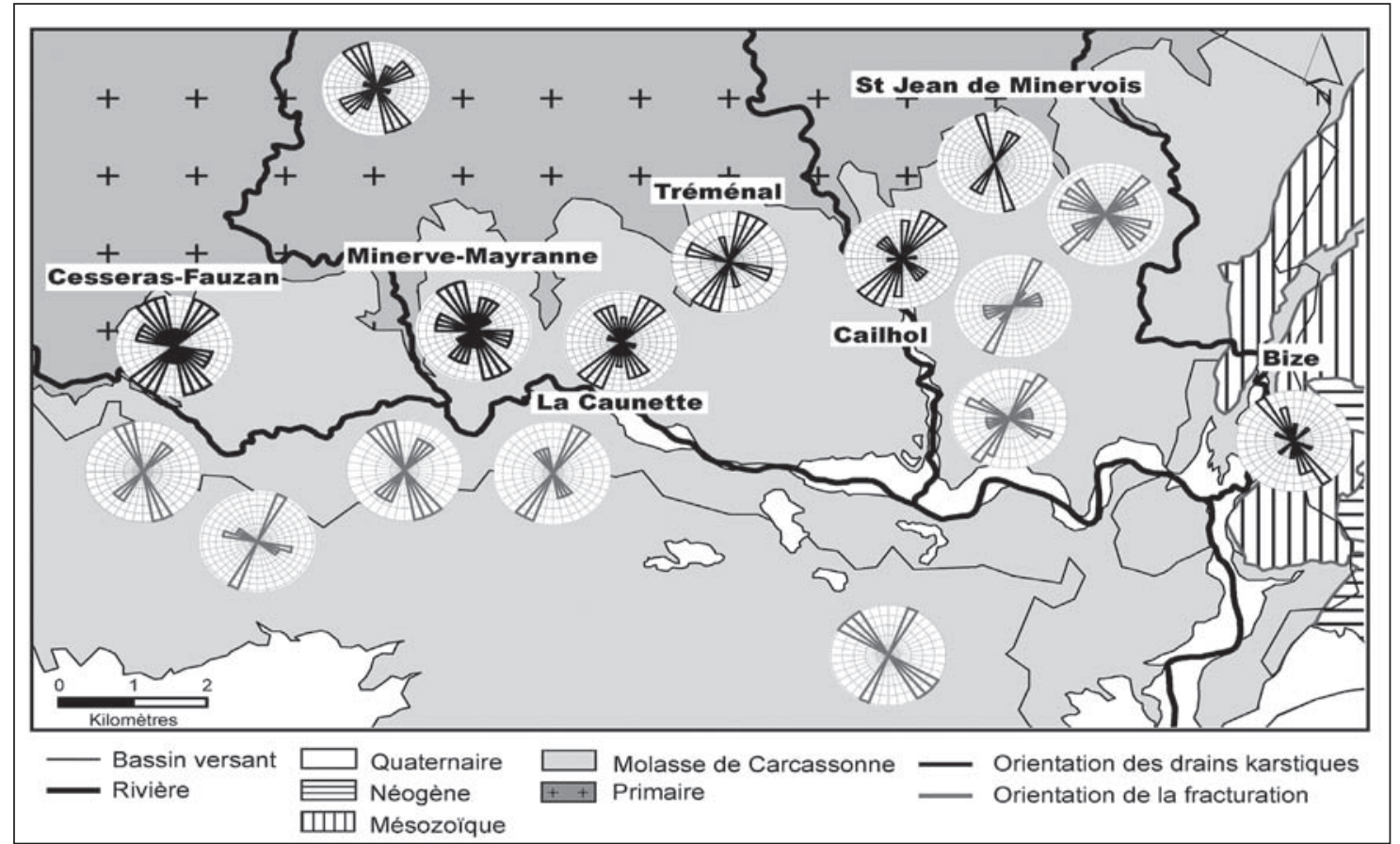

Fig. 2 : Distribution de la fracturation et des drains karstiques par rapport à leur longueur cumulée.

Fig. 2: Distribution of fractures and karstic drains based on their cumulative length. 


\section{3 - ÉVOLUTION HYDROGÉOLOGIQUE AU COURS DU QUATERNAIRE}

Pour la Cesse, deux ensembles de roches calcaires jouent un rôle prépondérant dans la formation de son réseau souterrain : les calcaires dolomitiques du Cambrien moyen et les calcaires éocènes. Dans la série tertiaire, les calcaires à Alvéolines (Ilerdien inférieur à moyen), les calcaires de Ventenac (Cuisien) et les calcaires d'Agel constituent les principaux niveaux réservoirs ainsi que le lieu des différentes résurgences. L'évolution du réseau souterrain à travers ces réservoirs est étroitement liée à celle du réseau hydrographique, ce qui implique de prendre en compte l'intégralité de la zone d'étude afin de proposer un scénario d'évolution de ces réseaux au cours du temps.

\section{1 - L'HYDROSYSTÈME KARSTIQUE DE LA CESSE ANTÉ-QUATERNAIRE}

Dans sa partie occidentale, le réseau hydrographique de la Cesse au Pliocène était très différent de sa configuration actuelle. Des terrasses alluviales, situées en aval de la source de Laval et datées du Pléistocène moyen, contiennent des éléments paléozoïques provenant de la Montagne Noire. La présence de ces éléments paléozoïques indique qu'avant le Pléistocène moyen, l'origine des réseaux hydrographiques de l'Ognon et de l'Espène se trouvait plus au nord, sur la Montagne Noire (Genna \& Capdeville, 2007). Le réseau hydrographique occidental actuel de la Cesse (en amont de Minerve), n'appartenait pas à cette dernière mais constituait la partie haute de l'Ognon et de l'Espène (fig. 3).

Le réseau souterrain karstique, actuellement associé à la partie occidentale de la Cesse, peut être interprété comme n'ayant pas été formé par la Cesse elle-même, mais par les eaux des réseaux hydrographiques de l'Ognon et de l'Espène qui s'écoulaient sur la Montagne Noire. La partie amont de ce réseau karstique, développé selon les directions principales de la fracturation de ce secteur, est constituée par les calcaires dolomitiques $\mathrm{du}$ Cambrien qui appartiennent au socle hercynien. Ces calcaires du Cambrien se situent entre Ferrals-lesMontagnes (source de la Cesse) et le secteur de Fauzan (zone de perte principale de la Cesse) et se structurent en trois bandes de puissance différente. Ces calcaires sont alimentés par les eaux qui ruissellent sur le socle primaire situé plus au nord. Les eaux souterraines qui les traversent sont alors i) cédées aux calcaires à Alvéolines lorsque ceux-ci les surmontent, ii) ramenées à la surface sous forme de résurgences au contact de roches imperméables (schistes ou grès) localisées plus au sud. Ce réseau karstique est toujours actif et alimente de nombreuses sources comme par exemple la source des Clauses (670 m), la source Saint-Pierre (450 m), la source d'Authèze $(440 \mathrm{~m})$. La partie aval du réseau karstique, également formée selon les directions principales de la fracturation de ce secteur, est constituée par les calcaires à Alvéolines sous couverture. Elle est comprise entre les accidents de Fauzan, l'amont du village de Minerve et la source de Laval qui est son exutoire. Le réseau karstique aval était alimenté soit par le réseau de surface (pertes

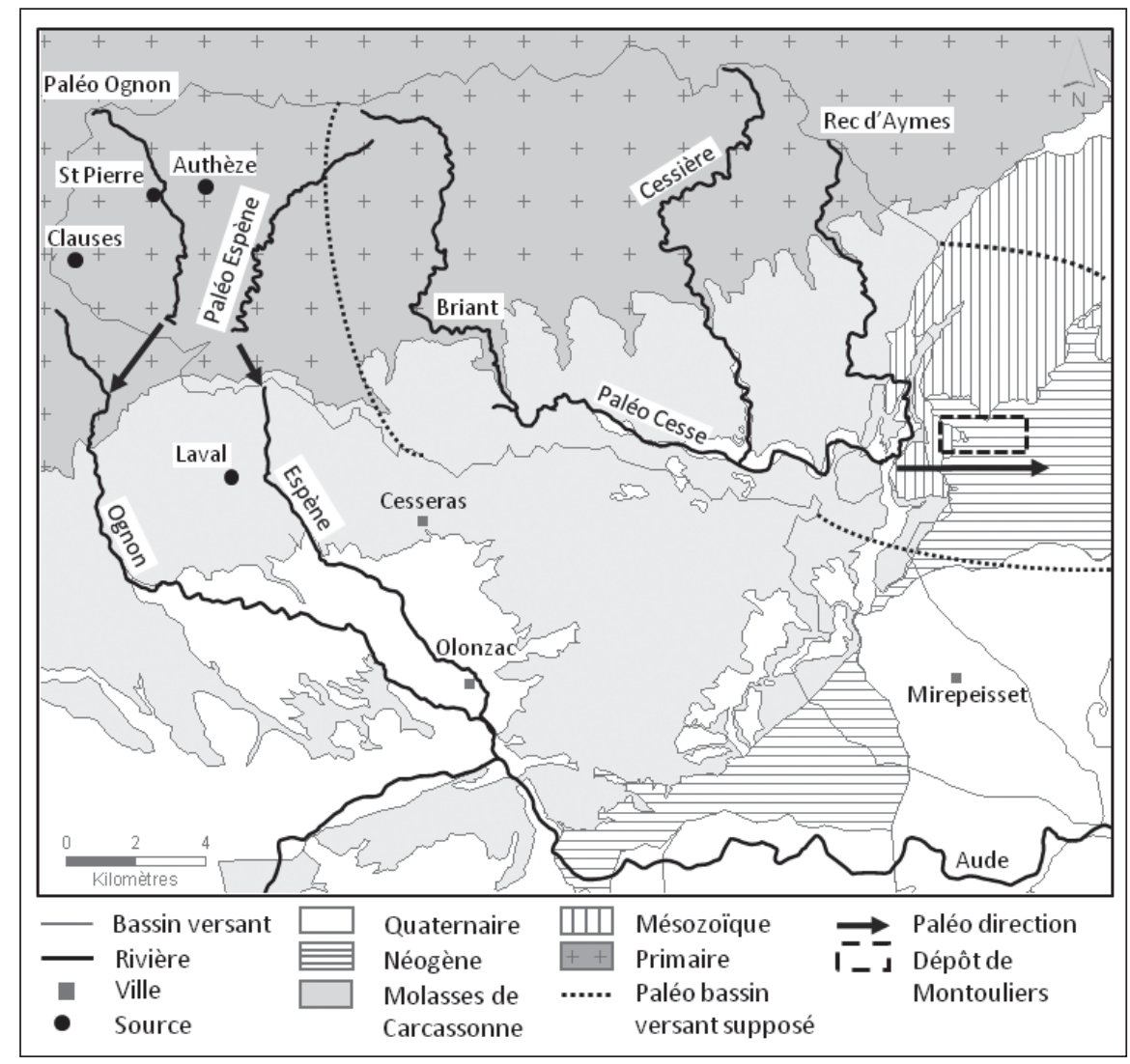

Fig. 3 : Paléo-hydrogéologie du bassin versant de la Cesse avant le Quaternaire.

Fig. 3: Paleo-hydrogeology of the Cesse watershed before the Quaternary. 
concentrées) soit par contact direct avec le réseau souterrain primaire. L'exutoire de ce système est représenté par la source de Laval (176 m) (fig. 3), avec un contact par faille entre les calcaires à Alvéolines (Ilerdien) et les formations d'Assignan (Cuisien) (Guerret, 1951; Genna \& Capdeville, 2007). Les nombreuses grottes qui se situent dans les calcaires à Alvéolines dans ce secteur du bassin, comme la grotte des Poteries $(325 \mathrm{~m})$, la grotte du Galzin $(305 \mathrm{~m})$, la grotte d'Aldène $(250 \mathrm{~m})$ et la grotte du chemin d'Azam (232 m), sont les témoins du réseau souterrain du paléo-Ognon et du paléo-Espène (fig. 3 ). Les formes endokarstiques ainsi que leurs remplissages (de taille centimétrique à décimétrique) traduisent la présence de débits souterrains importants.

Dans la partie orientale du bassin versant de la Cesse, contre les limites sud et est du bassin, par-dessus l'écaille de Montbrun (en butée contre la faille de Sainte-Valière) et contre le chaînon de Saint-Chinian, se sont déposés les terrains du Miocène marin et continental ainsi que du Pliocène continental (Larue, 2008). Le cours de la Cesse était différent du cours actuel. Au lieu d'emprunter la cluse de Bize sur une direction nord/sud, elle se poursuivait ouest/est en direction d'Argeliers et du fleuve l'Orb (fig. 3). La terrasse alluviale de Montouliers $(211 \mathrm{~m}$ NGF) en est le témoin (Le Coz, 1967; Berger, 1990; Larue, 2008). Le réseau souterrain de cette paléo-Cesse est similaire aux réseaux décris précédemment pour l'Ognon et l'Espène. Il se développe à la fois dans les terrains karstiques primaires du Cambrien et dans les calcaires à Alvéolines de l'Ilerdien selon les directions principales de la fracturation. Les deux systèmes sont reliés soit par contact direct soit par résurgence/infiltration. Les ponts naturels de Minerve, aujourd'hui occupés par le lit de la rivière en sont les traces. À la charnière Mio-Pliocène, débute une surrection de la bordure sud du Massif Central qui perdure jusqu'au Pliocène (Gèze, 1951; Ambert \& Ambert, 1995; Séranne et al., 2002; Larue, 2008). Cette surrection, couplée aux variations eustatiques liées à la crise Messinienne (Lofi et al., 2003 ; Larue, 2008) entraîne la capture de la Cesse au Pliocène supérieur par un ravin formé sur le versant sud du chaînon de Saint-Chinian et remontant le petit synclinal local compris entre le Mont Cayla et le pech de Bize (Gèze, 1951). Des terrasses alluviales dites de «Montplo» et datées du début du Pléistocène sont localisées à la côte de 190 m NGF au niveau de la terrasse de Montouliers ainsi qu'au niveau du synclinal de Pouzols à la côte de 116 m NGF (Le Coz, 1967) (fig. 4). Le cours de la Cesse passe d'ouest/est à nord/sud en direction de Bize, puis sur le synclinal de Pouzols avant de confluer avec l'Aude entre les villages de Paraza et de Ventenac. L'ancienne Cesse pliocène est alors asséchée pendant la période de transition par la résurgence de la grotte de Bize au travers des calcaires crétacés de la Verdeyre. Une fois la Cesse pliocène sèche, la résurgence de la grotte de Bize ainsi que son réseau, sont devenus fossiles.

La conséquence d'un abaissement du niveau de base associé au cours de la Cesse du début du Quaternaire peut être interprétée d'un point de vue hydrogéologique par une accentuation du drainage de l'anticlinal de la Serre d'Oupia qui a probablement pour conséquence la mise en place d'un exutoire certainement proche de la faille

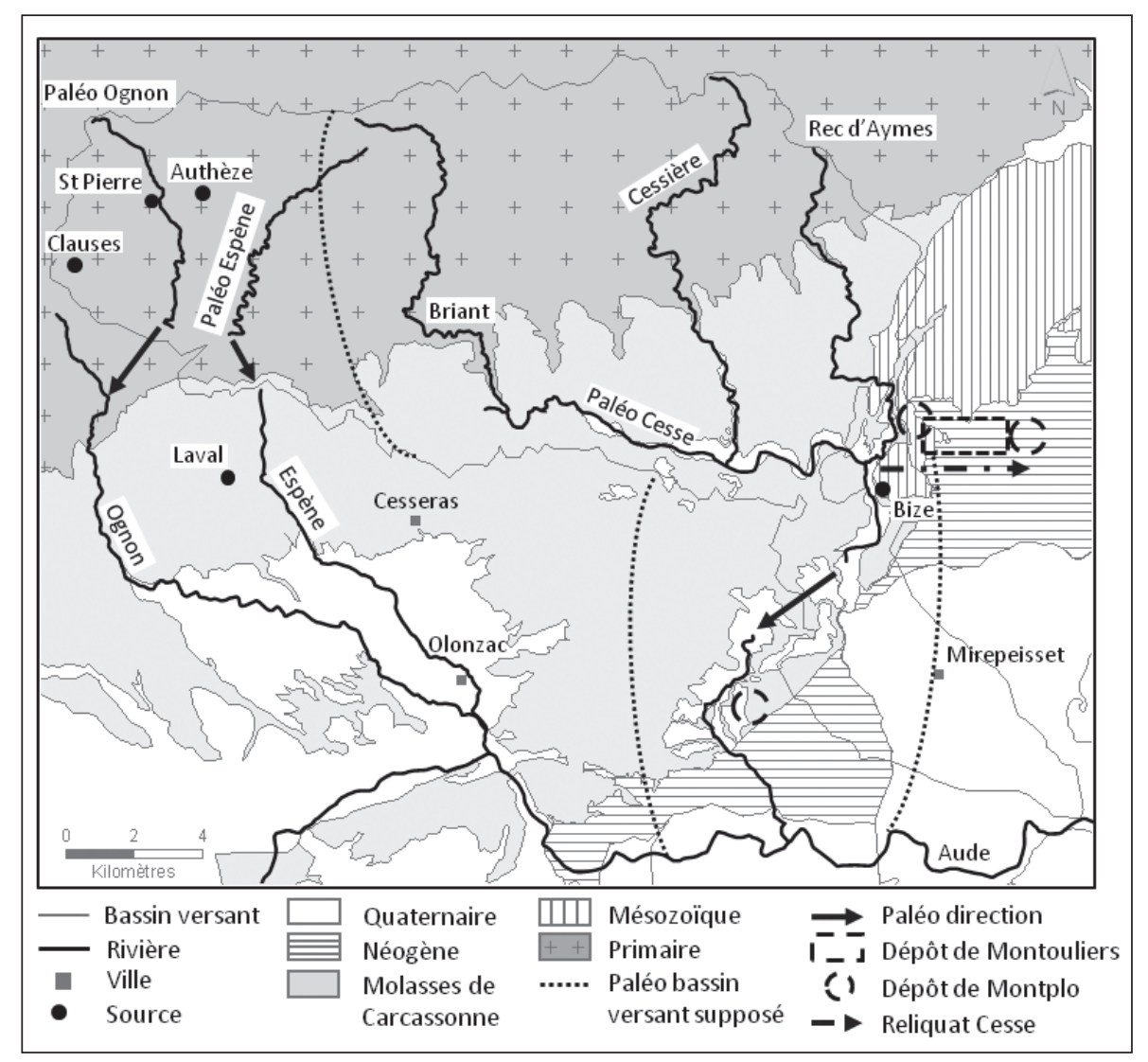

Fig. 4 : Paléo-hydrogéologie du bassin versant de la Cesse à la charnière Plio-Quaternaire.

Fig 4: Paleo-hydrogeology of the Cesse watershed during the Pio-Quaternary transition. 
de Sainte Valière et aujourd'hui abandonné. Cette accentuation du drainage a favorisé le développement d'une karstification profonde dans les calcaires de Ventenac du secteur du synclinal de Pouzols. Ceci est étayé par les résultats d'essais par pompage qui indiquent dans ce secteur de fortes transmissivités allant de $10^{-4}$ à $10^{-1} \mathrm{~m}^{2} / \mathrm{s}$. Dans la partie haute de la Cesse (de Minerve au chainon de Saint-Chinian), il permet également l'enfoncement du réseau souterrain précédent de la Cesse au niveau des fractures parallèles au gradient hydraulique. L'érosion induite par le gauchissement de la Montagne Noire et l'abaissement du niveau de base, entraîne également un accroissement de la surface d'affleurement des calcaires à Alvéolines au détriment de la couverture lacustre (calcaires de Ventenac).

\section{2 - LHYDROSYSTÈME KARSTIQUE DE LA CESSE AU QUATERNAIRE}

La basse vallée de la Cesse, avec sa confluence avec 1'Aude entre les communes de Paraza et de Ventenac, s'est maintenue jusqu'au Quaternaire moyen (Mindel) (Le Coz, 1967). Le dégagement de la couverture lacustre et l'enfoncement du réseau hydrographique ont été rapides pendant cette période. La Cesse recoupe dans sa partie amont son karst, mettant en place plusieurs résurgences comme la résurgence du petit pont de Minerve $(155 \mathrm{~m}$ $\mathrm{NGF}$ ) et la résurgence de la Doux de Coupiat (130 m NGF). Actuellement ces deux sources sont temporaires (fig. 5).
Au niveau du réseau karstique, le développement et l'enfoncement des eaux souterraines au sein des calcaires de Ventenac du synclinal de Pouzols se poursuit. Leur communication avec les calcaires à Alvéolines pourrait très certainement être établie dès cette période. La période post-mindélienne est marquée, en aval de Bize, par un enfoncement du lit des rivières [de l'ordre d'une vingtaine de mètres d'après Le Coz (1967)]. C'est à cette période que la Cesse a de nouveau changé de direction. Elle quitte la dépression de Mailhac-Pouzols pour se diriger, après Cabezac, vers Mirepeisset. Des terrasses du Riss et du Würm se trouvent aux altitudes de 50 m NGF et $40 \mathrm{~m}$ NGF en direction de Mirepeisset après Cabezac (Le Coz, 1967) (fig. 6). La confluence entre la Cesse et l'Aude s'établit alors sur la commune de Sallèles d'Aude qui est la confluence actuelle. Ce nouvel enfoncement a pour première conséquence l'apparition d'une nouvelle ligne de résurgences dans le lit de la rivière (Boulidou d'Agel: 75 m NGF, source de la Font de Pal : 49 m NGF, source de la Chaussée: $47 \mathrm{~m}$ NGF) dans le secteur de Bize (entre Agel et Cabezac). Ces sources drainent les calcaires de Ventenac à travers quelques mètres d'alluvions, ce qui est rendu possible par l'accentuation du débit souterrain liée à la dénudation des calcaires situés en amont.

La deuxième conséquence de l'enfoncement de la Cesse dans sa basse vallée, d'après Genna \& Capdeville (2007), est une érosion régressive de la partie amont de son réseau hydrographique. Cette nouvelle observation a pour conséquence la capture en rive gauche de la

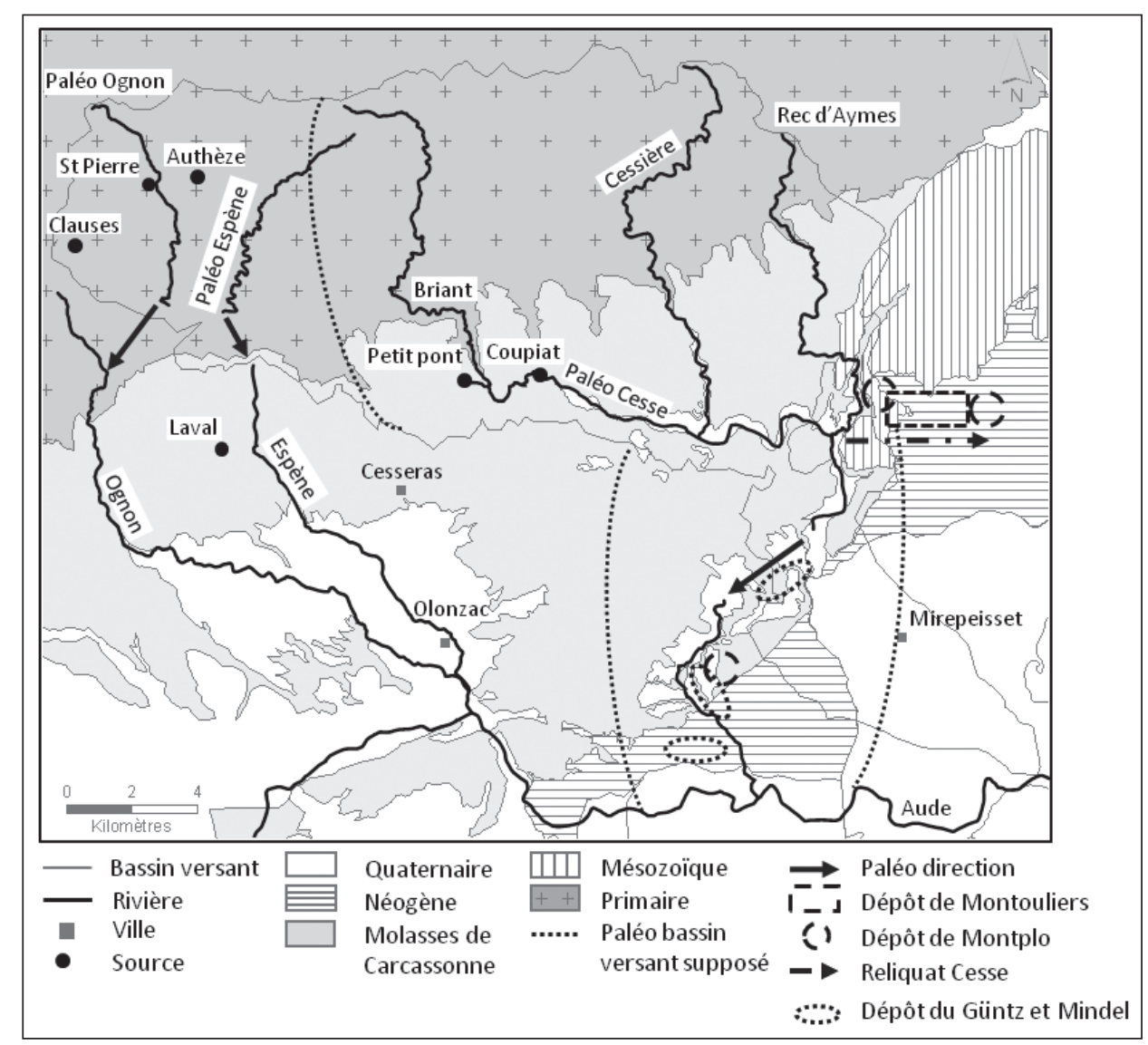

Fig. 5 : Paléo-hydrogéologie du bassin versant de la Cesse au Quaternaire moyen.

Fig. 5: Paleo-hydrogeology of the Cesse watershed during the middle of Quaternary. 
partie amont de l'Espène et de l'Ognon, ce qui indique que c'est seulement au cours de la deuxième partie du Quaternaire que la Cesse a acquis son réseau hydrographique complet et actuel (fig. 6). Dans la partie amont, la capture du réseau hydrographique amont de l'Espène et de l'Ognon a également entraîné la capture de leur réseau karstique. Le réseau karstique entre Fauzan et l'amont de Minerve a alors été annexé au réseau de la Cesse. Les écoulements souterrains nord/sud, sont passés globalement ouest-nord-ouest/sud-sud-est et ouest/est à la faveur des joints de stratification alors parallèles au nouveau gradient hydraulique. Ce phénomène de capture pourrait expliquer les directions des drains parallèles au cours de la Cesse dans le secteur de Cesseras-Fauzan et de Minerve Mayranne, alors qu'aucune fracture orientée dans cette direction n'a été observée dans ces secteurs ( 33.1 ; fig. 2). Une partie des eaux souterraines a tout de même gardé sa direction d'origine vers la source de Laval, pérenne de nos jours mais désormais alimentée par une petite partie des pertes de la Cesse. La flexure du Minervois a probablement joué un rôle essentiel, lorsque la Cesse a capturé les deux cours d'eau en surface, en facilitant la capture du réseau souterrain (fig. 7). C'est dans ce secteur, complexe au niveau de son évolution hydrogéologique, qu'une morphologie de drains comparable à celle de karsts hydrothermaux a été observée avec une karstification labyrinthique observée sur la discordance entre les dolomies cambriennes et les calcaires à Alvéolines de l'Ilerdien (Nou et al., 2010). Cependant, il est probable que la morphologie de ces drains ne soit pas directement reliée à la présence d'un karst hydrothermal mais témoignerait de la capture d'un réseau karstique par un autre. Cette morphologie est très remarquable en amont du pont naturel de Minerve, en rive gauche du lit de la Cesse, à peine au-dessus des alluvions récentes.

Les directions des drains des deux réseaux karstiques sont différentes, globalement nord-sud pour les réseaux de l'Ognon et de l'Espène et ouest-est pour le réseau amont de la Cesse. Le passage d'une direction préférentielle à l'autre sur ce secteur peut expliquer l'organisation labyrinthique des drains karstiques observée qui passent d'une direction à l'autre. Le haut niveau piézométrique nécessaire à cette organisation ainsi qu'à la forme en dôme des drains, peut être expliqué par la flexure du Minervois pouvant être associée à une barrière semi-perméable. Dans sa partie aval, le réseau souterrain karstique de la Cesse a été modifié. La karstification, probablement profonde, a été essentiellement développée dans le synclinal de Pouzols par la paléo-Cesse du début Quaternaire selon un axe nord/sud. Lors de l'enfoncement et de la dernière défluviation de la Cesse, l'apparition des sources pérennes entre Agel et Cabezac a déplacé les exutoires du karst dans sa partie orientale directement dans le lit de la rivière. Le karst du synclinal de Pouzols est donc un karst probablement profond et barré par la faille de Sainte-Valière. Cette dernière sert de limite entre les terrains karstiques de l'Éocène du synclinal de Pouzols et les terrains mio-pliocènes étanches. Sa partie la plus méridionale, principalement karstifiée au début du Quaternaire, se retrouve de nos jours plus au sud que

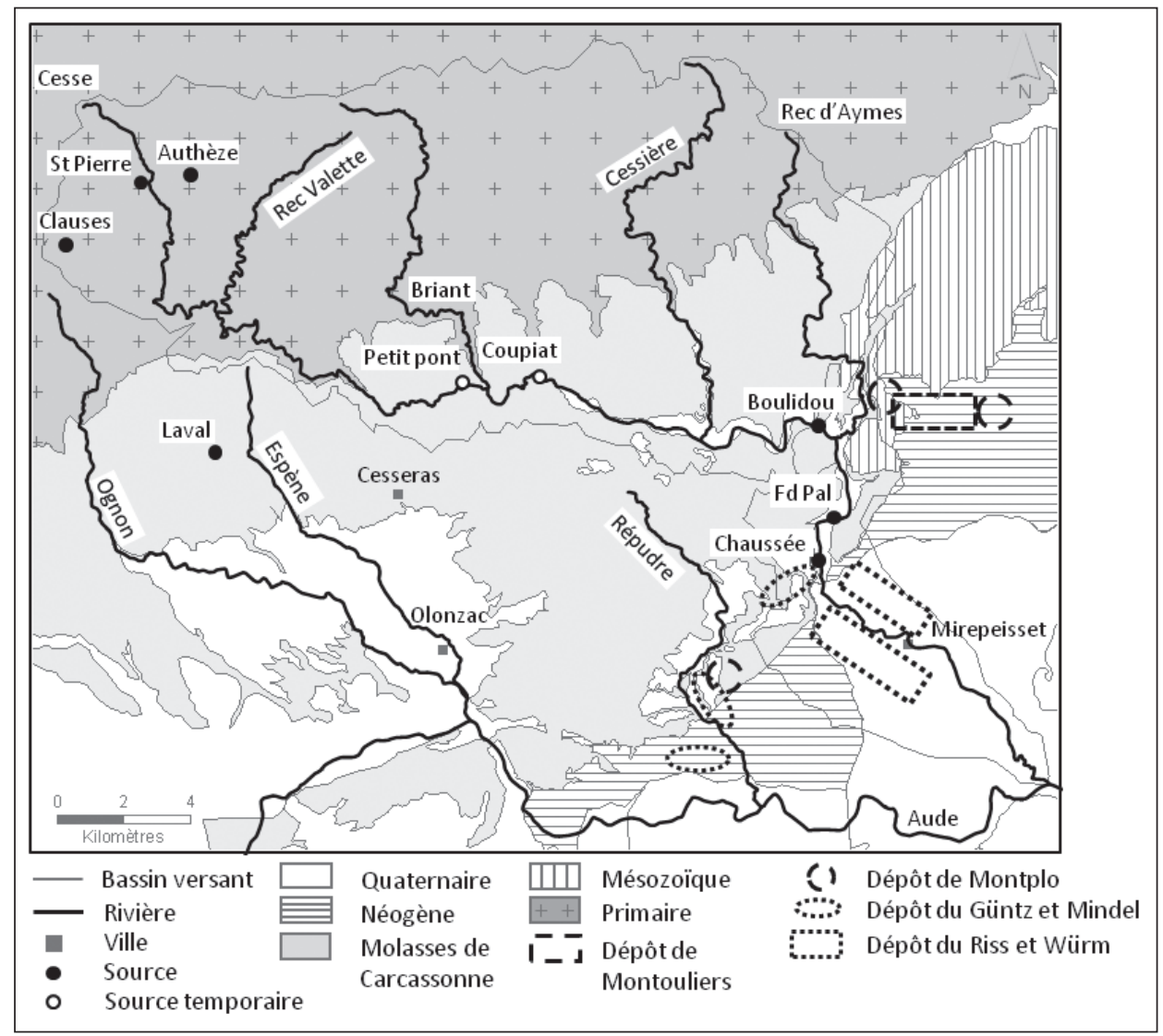

Fig. 6 : Hydrogéologie actuelle du bassin versant de la Cesse.

Fig. 6: Present hydrogeology of the Cesse watershed. 


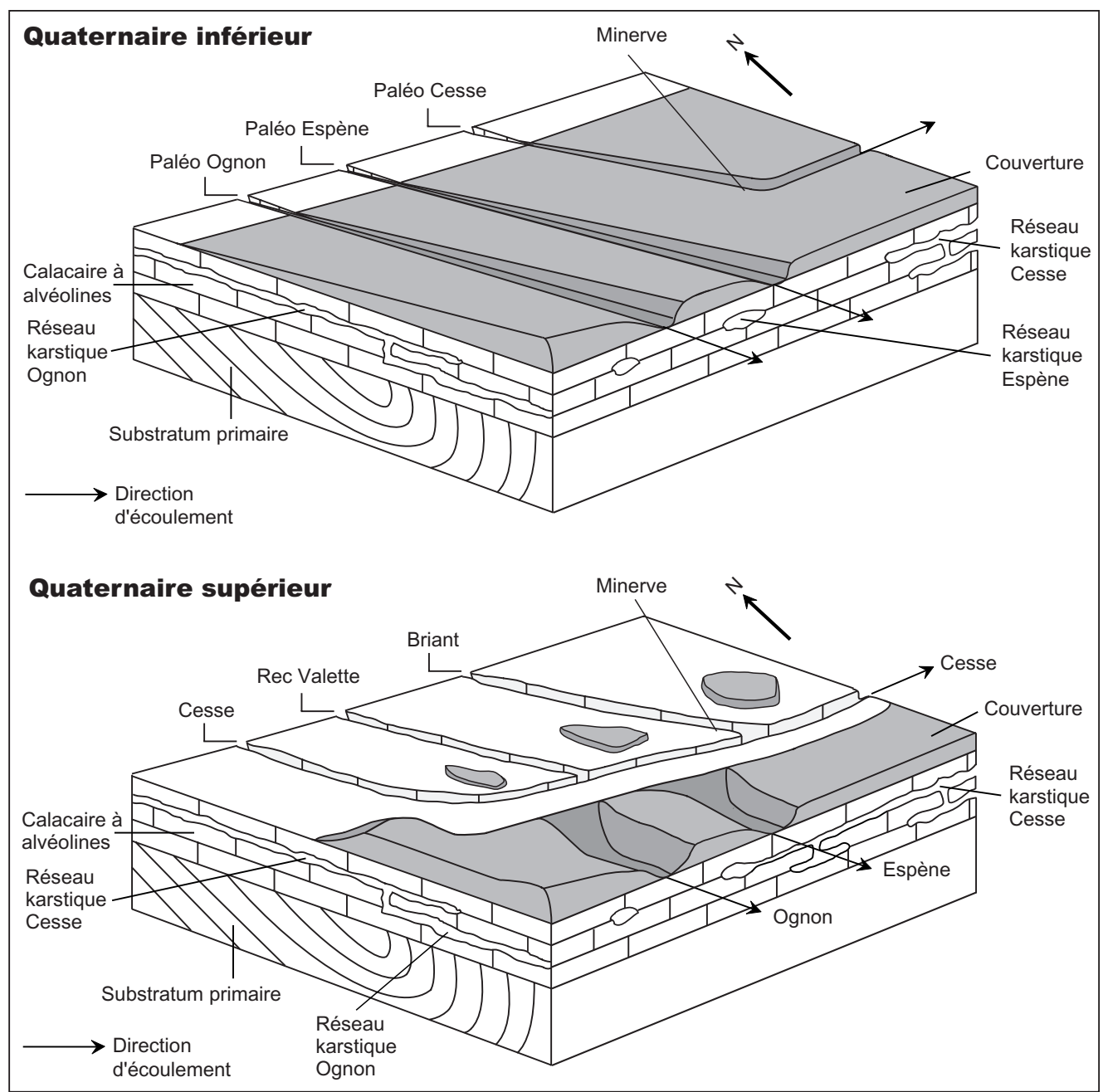

Fig. 7 : Bloc-diagramme synthétique du phénomène de capture des réseaux karstiques par l'évolution du réseau hydrographique au cours du Quaternaire.

Fig. 7: Schematic block diagram describing the karstic network capture by the evolution of the hydrographic network.

ses exutoires actuels. Au vu de la structure géologique et de l'extension nord-est de la faille de Sainte-Valière, il est donc probable que la totalité du karst du synclinal de Pouzols soit en relation avec les exutoires actuels. La faille de Sainte-Valière permettant une connexion hydraulique entre la partie la plus méridionale et les sources de la Cesse.

\section{4 - CONCLUSION}

L’hydrosystème karstique méditerranéen CessePouzols est constitué par deux grands ensembles géologiques distincts: les terrains primaires du versant sud de la Montagne Noire et les terrains tertiaires de l'Éocène en discordance sur ces derniers. Sur la partie amont $\mathrm{du}$ bassin, les eaux ruissellent sur les terrains de la Montagne Noire ou sont infiltrées dans les systèmes karstiques cambriens qui se développent le long de la fracturation. Cette partie du bassin peut être alors considérée comme l'aire principale d'alimentation du bassin. Au contact des calcaires à Alvéolines de l'Ilerdien, les eaux de surface se perdent dans le sous-sol ou leur sont cédées. Elles contribuent ainsi à l'alimentation de la partie amont de l'aquifère de Pouzols. Cette partie de l'aquifère, qui comprend le réseau karstique propre à la Cesse ainsi que celui capturé à la partie amont de l'Espène et de l'Ognon se développe le long de la fracturation mais également le long des joints de stratification, principalement dans le secteur de Minerve. Elle constitue également la zone non saturée de l'aquifère où se trouvent les sources temporaires du petit pont de Minerve et de la Doux de Coupiat. La circulation des eaux souterraines suit ici une direction globalement ouest/est conditionnée dans la partie occidentale par la flexure du Minervois (fig.8 ). Dans la partie orientale du bassin ces eaux ont une direction générale nord/sud, en direction des exutoires de l'aquifère de Pouzols. Dans leur parcours souterrain, les eaux contenues dans les calcaires à Alvéolines sont transmises aux calcaires de Ventenac (Cuisien) généralement à la faveur d'un contact par faille et dans la partie la plus orientale du bassin les eaux souterraines peuvent aussi être cédées aux calcaires d'Agel (Lutétien). À la faveur de la karstification développée lorsque la Cesse occupait le synclinal de Pouzols au début du quaternaire, une partie des eaux en provenance de la partie occidentale du bassin alimente les calcaires de Ventenac du synclinal de Pouzols ainsi que les eaux infiltrées sur la bordure est de l'anticlinal 


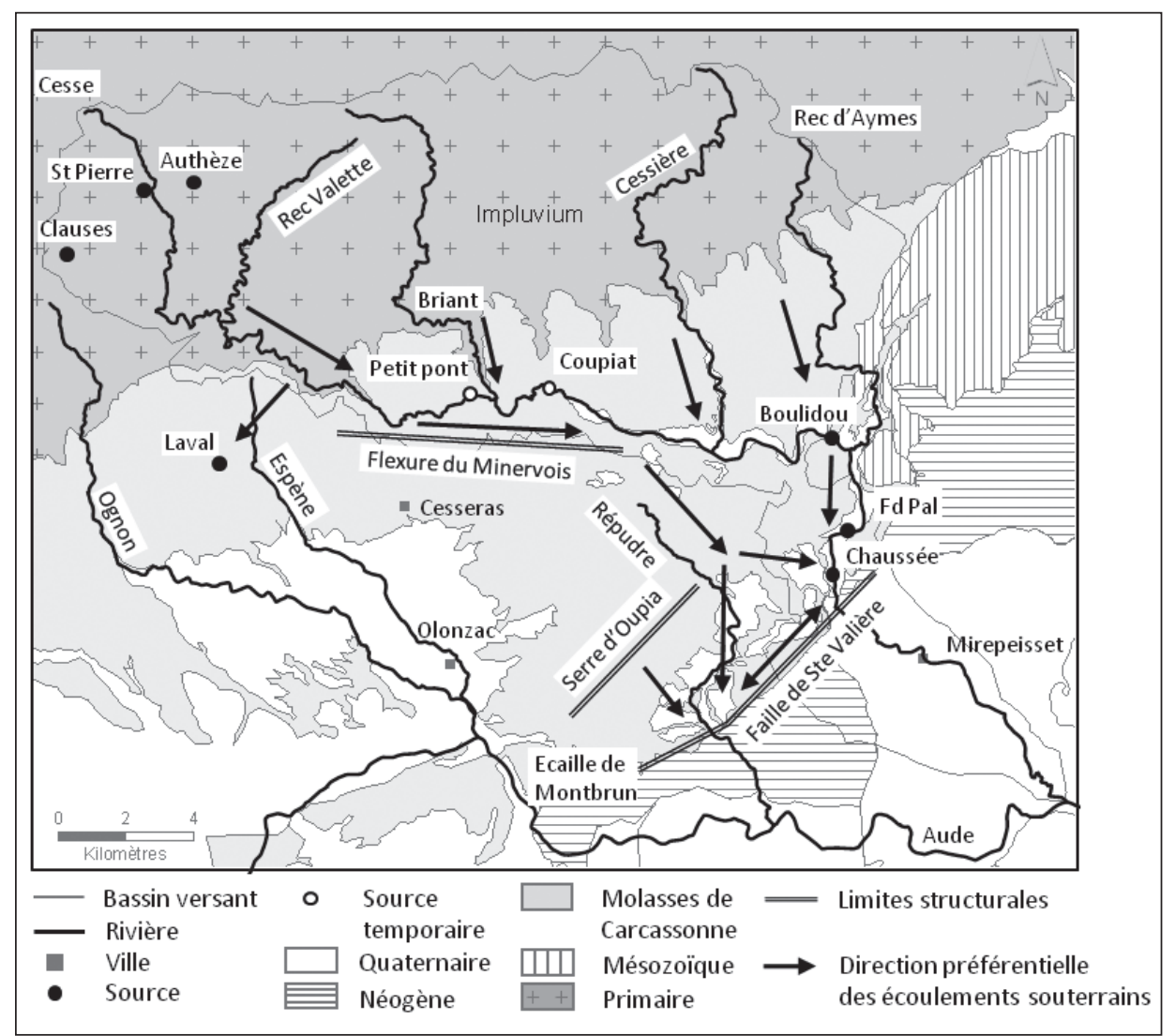

Fig. 8: Schéma de fonctionnement de l'hydrosystème karstique Cesse-Pouzols.

Fig. 8: Functioning sketch of the Cesse-Pouzols karst hydrosystem.

de la Serre d'Oupia. Ces eaux sont bloquées au sud par la faille de Sainte-Valière et l'écaille de Montbrun qui leur opposent les terrains imperméables du Mio-Pliocène pour la première et une probable limite structurale étanche pour la deuxième (fig. 8).

L'hydrosystème karstique Cesse-Pouzols peut être résumé en trois unités :

(i) l'impluvium, constitué des terrains primaires de la Montagne Noire,

(ii) une grande zone non saturée libre, constituée des calcaires à Alvéolines, des calcaires de Ventenac des causses du Minervois ainsi que de la Serre d'Oupia et des calcaires d'Agel lorsqu'ils affleurent,

(iii) une vaste zone noyée captive dont les limites générales sont: la faille de Sainte-Valière au sud et au sud-est, le chaînon de Saint-Chinian à l'est, la résurgence du Boulidou d'Agel au nord-est et l'anticlinal de la Serre d'Oupia à l'ouest (fig. 8).

La synthèse pluridisciplinaire des travaux antérieurs, associée à de nouvelles données d'orientation de la fracturation et des drains karstiques, ont permis de proposer un scénario cohérent d'évolution hydrogéologique de l'hydrosystème Cesse-Pouzols depuis la fin du Tertiaire jusqu'à aujourd'hui. Elle s'appuie sur des interprétations et sur des hypothèses étayées par des observations directes d'ordre structural ou hydrodynamique complétant les résultats antérieurs. La reconstitution de l'histoire du bassin versant de la Cesse d'un point de vue des interactions surface/souterrain est fondamentale pour établir un schéma de fonctionnement hydrogéologique global afin d'améliorer la compréhension et la gestion d'une ressource exploitée. La zone noyée, localisée dans le synclinal de Pouzols, est majoritairement captive et d'extension importante. Elle peut donc représenter une réserve importante non négligeable d'eau, en permanence alimentée par les pertes de la Cesse et ses affluents. Néanmoins le comportement hydrodynamique ainsi que le temps de séjour des eaux souterraines de cet hydrosystème en cours d'exploitation restent encore mal connus.

\section{REMERCIEMENTS}

Les auteurs remercient le spéléo-club de Béziers et notamment son président M. Jacky Fauré pour son aide active afin d'accéder à l'inventaire en cours de finalisation et aux cavités remarquables qui y sont mentionnées. Le conseil Général de l'Aude est aussi remercié pour son soutien technique lors de cette étude.

\section{RÉFÉRENCES BIBLIOGRAPHIQUES}

AMBERT P., 1976 - Les ponts naturels de Minerve (Hérault), étude géomorphologique. Méditerranée, 2, 43-53.

AMBERT P., AMBERT M. \& MAURIN G., 1977 - L'évolution du remplissage de la grotte des Poteries (Cesseras-Hérault). Spelunca. Bulletin, 1977 (3), 125-127. 
AMBERT M. \& AMBERT P., 1995 - Karstification des plateaux et encaissement des vallées au cours du Néogène et du Quaternaire dans les Grands Causses méridionaux (Larzac, Blandas). Géologie de la France, 4, 37-50.

ARTHAUD F., 1964 - Étude structurale de la terminaison occidentale des Monts de Faugères (Montagne Noire). Bulletin des Services de la Carte Géologique de France, 277, 133-145.

BERGER G.M., 1990 - Carte géologique de la France à 1/50000, Lézignan-Corbières (1038). Notice explicative par BERGER G.M., BOYER F., REY J., avec la collaboration de AMBERT P., FREYTET P., MARCHAL J.P. \& VAUTRELLE C., 1990. Bureau de Recherches Géologiques et Minières, Orléans, 70 p.

BERTOLINI P., 1980 - Étude des aquifères karstiques de la région «Saint-Pons, Minerve». Structure des magasins, organisation des écoulements souterrains. Thèse de Doctorat, Université Montpellier 2, Montpellier, $117 \mathrm{p}$.

CHAIGNE M., 1964 - Contribution à l'étude stratigraphique et sédimentologique du secteur Aigne-Tourouzelle, bassin tertiaire de Carcassonne, Aude. Thèse de Doctorat, Université de Bordeaux, Bordeaux, $116 \mathrm{p}$.

CORNET C., 1977 - Les surfaces d'arasion des Corbières. Compte Rendu Sommaire des Séances de la Société Géologique de France, 1, 7-8.

GENNA A., 1989 - Relations entre tectonique cassante, déformation hydroplastique et circulation de fluide dans l'extrémité Est du bassin Eocène de Carcassonne. Thèse de Doctorat, Université Montpellier 2, Montpellier, $197 \mathrm{p}$.

GENNA A. \& CAPDEVILLE J.-P., 2007 - Réorganisations hydrographiques du Minervois, exemples de la Cesse et du ruisseau du SaintMichel (Hérault, France) au Quaternaire, conséquences géologiques. Quaternaire, 18 (3), 271-282.

GÈZE B., 1951 - Sur la probabilité d'un gauchissement quaternaire important à la bordure méridionale de la Montagne Noire (Aude, Hérault). In Association Française pour l'Avancement des Sciences (éd.), $70^{e}$ congrès de l'Association Française pour l'Avancement des Sciences: comptes rendus et communications, Tunis, 9-16 mai 1951. Fascicule 1, Sections de mathématiques, mécanique, génie civil, météorologie et physique du globe, géologie et minéralogie, géographie. Nicolas Bascone \& Sauveur Muscat imprimeurs, Tunis, 108-110.
GUERRET M., 1951 - $A$ travers la Montagne noire: la Cesse minervoise, pertes et résurgences. J. Busson, Montauban, $40 \mathrm{p}$.

LARUE J.-P., 2001 - Tectonique et dynamique fluviale quaternaires: l'exemple de la basse vallée de l'Aude (France). Quaternaire, 12 (3), 169-178.

LARUE J.P., 2008 - Effects of the tectonics and lithology on long profiles of 16 rivers of the southern Central Massif Border between the Aude and the Orb (France). Geomorphology, 93 (3-4), 343-367.

LE COZ J., 1967 - Aspects du Quaternaire languedocien: les niveaux de la Cesse et de l'Orbieu (Aude). Bulletin de la Société Languedocienne de Géographie, 1 (2), 127-146.

LOFI J., RABINEAU M., GORINI C., BERNE S., CLAUZON G., DE CLARENS P., TADEU DOS REIS A., MOUNTAIN G.S., RYAN W.F., STECKLER M.S. \& FOUCHET C., 2003 - PlioQuaternary prograding clinoform wedges of the western Gulf of Lion continental margin (NW Mediterranean) after the Messinian Salinity Crisis. Marine Geology, 198 (3-4), 289-317.

NOU A., PISTRE S., BORRELL-ESTUPINA V., BATIOT-GUILHE C., BAKALOWICZ M., 2010 - Evidence of an Early Phreatic and Confined Karst Phase in Minervois, South of France. In B. Andreo, F. Carrasco \& J.J. Duran (eds.), Environmental Earth Sciences, Advances in Research in Karst Media. Springer, London, 526 p.

ORENGO R., 1969 - Étude bibliographique et hydrogéologique de la région du Minervois (Aude et Hérault). Rapport d'étude, Université Montpellier 2, Montpellier $41 \mathrm{p}$.

SÉRANNE M., CAMUS H., LUCAZEAU F., BARBARAND J. \& QUINIF Y., 2002 - Surrection et érosion polyphasées de la bordure cévenole. Un exemple de morphogenèse lente. Bulletin de la Société Géologique de France, 173 (2), 97-112.

YVROUX M., 2001 - L'aquifère karstique de Pouzols-Minervois (Aude, France). Synthèse hydrogéologique, Conseil Général de l'Aude, Carcassonne, 73 p. 